\title{
Cutaneous nerve stimulation and motoneuronal excitability. II: evidence for non-segmental influences
}

\author{
PJ DELWAIDE, P CRENNA* \\ From the Section of Neurology and Clinical Neurophysiology University of Liège, Liège, Belgium
}

SUMMARY After stimulation of a sensory nerve, even distant motor nuclei undergo excitability changes which are evidenced by recovery curves. In all, two unequal peaks of facilitation can be identified. They appear when a given motor nucleus is tested after stimulation of various sensory nerves or when the same stimulation (of the sural nerve) is studied in various motor nuclei. The first facilitation is seen earlier in the masseter, then in the arm muscles and finally in lower limb muscles. The existence of a supraspinal centre activated by low threshold exteroceptive afferents and facilitating motor nuclei in a rostro-caudal sequence is postulated to account for certain features of the first and second peaks of facilitation.

In an earlier publication, we ${ }^{1}$ described excitability changes in soleus and tibialis anterior motoneurons following stimulation of the sural nerve at different intensities. A stimulus intensity which elicits tactile sensation $(2.5 \times$ perception threshold $)$ increased the amplitude of test monosynaptic soleus reflexes over two successive periods, from 55 to $90 \mathrm{~ms}(\mathrm{Fa})$ and from 130 to $170 \mathrm{~ms}(\mathrm{Fb})$. Similar results were obtained when the sural nerve contralateral to the test reflex was stimulated.

The similarity of the results obtained after ipsilateral and contralateral stimulation is difficult to interpret on the basis of segmental spinal phenomena, and raises the question as to whether this double facilitation can also be elicited by stimulation of other sensory nerves. In such a case, the operation of a common but non-segmental mechanisms could be postulated; subsequently facilitation could also be observed distant from the segments in which exteroceptive afferents enter the cord. In the present work we have therefore investigated the effect on the soleus motor nucleus of stimulation of various sensory nerves: saphenous, lateral femoral cutaneous, iliohypogastric, median, and trigeminal. Subsequently we have studied the effect of identical

*From Istituto di Fisiologia Umana II, Universita degli Studi di
Milano, Milano (Italy).

Address for reprint requests: PJ Delwaide, MD, Section of Neurology, Bol de la Constitution 66, 4020 Liège, Belgium.

Received 28 December 1982 and in revised form 11 June 1983. Accepted 2 August 1983 sural nerve stimulation on various motor nuclei located along the length of the neuraxis: soleus, quadriceps, short biceps, biceps brachii and masseter.

\section{Subjects and Methods}

Seventeen normal volunteers of both sexes were studied, some of them several times; they were aged between 18 and 29 with a mean of 24.2 years. A detailed description of the equipment used, and the methods employed was given in an earlier publication.' Mention will only be made here of the technical variants used in the present study. In order to measure latencies more accurately, the duration of the conditioning stimulation was shortened to two onemillisecond shocks delivered at a frequency of $400 \mathrm{~Hz}$. Stimuli were repeated in a random manner in a time bin between 7.5 and 9.5 seconds. Stimulation of sensory nerves was effected by DISA $13 \mathrm{~K} 62$ cutaneous electrodes applied over the course of the nerves as follows: sural nerve-at the level of the external malleolus, saphenous nerve-over the medial aspect of the knee joint, lateral cutaneous nerve of thigh-at the anterior superior iliac spine, iliohypogastric nerve (posterior branch)-3 $\mathrm{cm}$ lateral to the spine of the first lumbar vertebra, supraorbital nerve $\left(\mathrm{V}_{1}\right)-1 \mathrm{~cm}$ above the supraorbital ridge.

The interdigital nerves of the 2nd and 3rd fingers, which are branches of the median nerve, were stimulated by ring electrodes slipped over the fingers. Unless otherwise stated the stimulus intensity was adjusted so as to elicit a painless tactile sensation; this normally corresponded to 2-3 times the sensory perception threshold (Thr).

The soleus motor nucleus was tested by means of the Hoffmann reflex elicited by the method described in Human Reflexes, vol. III. The motor nuclei of quadriceps and of the short biceps fernoris were investigated by means 
of their respective tendon reflexes. These latter were elicited by a Bruel and Kjaer vibrator solidly fixed opposite the tendon with the knee joint at an angle of $120^{\circ} \mathrm{A}$ No 4809 Bruel and Kjaer vibrator was also used to elicit biceps and triceps brachii tendon reflexes, (with a maximal displacement of $8 \mathrm{~mm}$ and a delay of $2 \mathrm{~ms}$ ) with the elbow joint fixed at $135^{\circ}$. A No. $4810 \mathrm{BK}$ vibrator, rigidly fixed in a mechanical holder, was used to percuss the mandible in the midline $1 \mathrm{~cm}$ below the lower lip with the mouth halfopen. The slightly extended head rested against a firm surface.

Cutaneous electrodes were fixed respectively: quadriceps: one at the upper border of the patella and the other $3 \mathrm{~cm}$ higher in the anterior midline of the thigh, short head of bicep femoris: one on the tendon in the upper part of the popliteal fossa, and the other $3 \mathrm{~cm}$ higher on the belly of the muscle, biceps brachii: one on the tendon at the level of the elbow and the other $3 \mathrm{~cm}$ higher, triceps brachii: one on the tendon $2 \mathrm{~cm}$ above the olecranon and the other $3 \mathrm{~cm}$ higher, masseter: one over the insertion of the muscle and the other $2 \mathrm{~cm}$ higher over the muscle.

For the $\mathrm{H}$ reflex and the limb tendon reflexes, the mean value of $\mathbf{3 0}$ responses was taken under control conditions and of at least 10 responses at each delay after conditioning. For the jaw jerk, at least 30 responses were averaged. The standard deviations of the control values were measured. Delays from 10 to $1000 \mathrm{~ms}$ were explored. During the first $200 \mathrm{~ms}$, measurements were made every $10 \mathrm{~ms}$, and sometimes every $5 \mathrm{~ms}$ when certain critical latencies were being explored. After $200 \mathrm{~ms}$, measurements were made at fewer time intervals. To determine the onset of a facilitation period the delay at which the amplitude of the mean value of the conditioned reflex intersects the standard deviation line of controls has been considered.

\section{Results}

\section{a. CHANGES IN SOLEUS MOTOR NUCLEUS EXCITABILITY ELICITED BY STIMULATION OF VARIOUS SENSORY NERVES}

Figure 1 is an illustrative example of the results obtained, all from the same volunteer subject. The intensities of stimulation used elicited a painless sensation of touch, and were applied on the same side as the soleus nucleus under test. Sural nerve stimulation $(2.2 \times \mathrm{Thr})$ caused the appearance of a facilitation characterised by two unequal peaks, as has been described previously. ${ }^{1}$ When the saphenous nerve was stimulated $(2.5 \times \mathrm{Thr})$, a double-peaked facilitation was again observed; it can be seen on the graph that the onset latency of the first peak was shorter than in the case of sural nerve stimulation. Account must obviously be taken of the conduction time between the site of the stimulation and the spinal cord. The amplitude of facilitation seen after saphenous nerve stimulation is about the same as that which follows sural nerve stimulation. Stimulation of the lateral cutaneous nerve of the thigh $(2 \cdot 5$ $\times \mathrm{Thr}$ ) also elicits two successive phases of facilitation, in which the first peak appears even earlier than in the two preceding cases; and in this particular instance it is of even greater amplitude.

The similarity between the three curves so far described should be emphasised, the principal difference being the successively earlier appearance of the first peak at approximately 58, 43 and $37 \mathrm{~ms}$ respectively.

Figure 1D shows the result of stimulating a sensory nerve in the ipsilateral lumbar region of the trunk rather than the lower limb. Following such stimulation $(2.3 \times \mathrm{Thr})$, the soleus motor nucleus again presents excitability changes which resemble those seen in fig $1 \mathrm{~A}-\mathrm{C}$; there are two peaks, which are however less marked than those following limb nerve stimulation. Thus the excitability of the soleus motor nucleus is modulated by stimulation applied to a sensory nerve outside the lower limb. Starting from these observations, stimulation was applied to the interdigital sensory branches of the median nerve. Here again, a two-peaked facilitation of soleus motor nucleus excitability was seen, at intensities similar to those which follow sural nerve stimulation $(2 \cdot 4 \times \mathrm{Thr})$. Thus it can be seen that a sensory nerve entering the cervical enlargement exerts an effect on a motor nucleus in the lumbar enlargement. Figure 1F shows excitability changes following stimulation $(2.4 \times \mathrm{Thr})$ of a branch of the first division of the trigeminal nerve. Once again, a double-peaked facilitation was observed. When the intensity of stimulation was progressively increased (see figure 2 where the exploration has been limited to intervals shorter than $100 \mathrm{~ms}$ and where a linear time-scale has been used), a moderate facilitation was seen for a stimulus intensity equal to twice the perception threshold. Facilitation amplitude increase progressively with stimulation at 2.4 and 3.8 times sensory perception threshold.

It can be seen in figs $1 \mathrm{D}$ to $\mathrm{F}$ that the latency of the first peak decreases progressively.

Observations similar to those illustrated in figs 1 and 2 were made in 12 subjects. The results confirmed that the soleus motor nucleus is regularly facilitated by stimulation of all these nerves, the mean onset latencies of the early facilitation being respectively: $56 \cdot 5 \pm 8 \mathrm{~ms}(\mathrm{SD})$ for sural nerve, $47 \cdot 2$ $\pm 13 \mathrm{~ms}(\mathrm{SD})$ for saphenous nerve, $40 \cdot 3 \pm 9 \mathrm{~ms}$ (SD) for lateral cutaneous nerve of the thigh, 71.4 \pm $12 \mathrm{~ms}$ (SD) for ileohypogastric nerve, $60 \cdot 2 \pm 10 \mathrm{~ms}$ (SD) for median and $61.8 \pm 11 \mathrm{~ms}$ (SD) for trigeminal nerve conditioning. There are however some differences according to the nerve stimulated. Following median nerve stimulation, a single peak of facilitation may be observed rather than a double peak such as that illustrated in fig 1 . On the other 


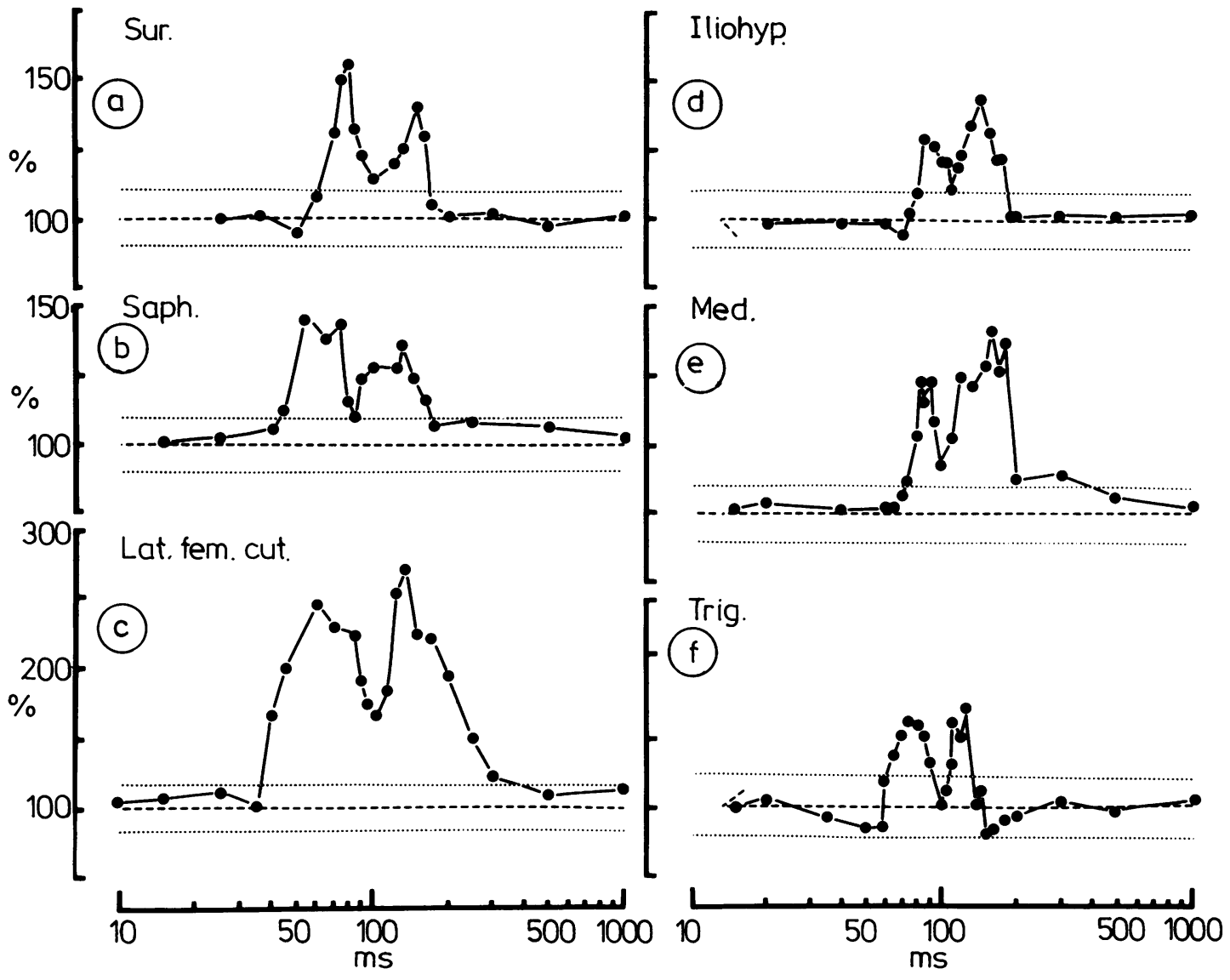

Fig 1 Recovery curves of the soleus $H$ reflex following conditioning stimulations of various sensory nerves: $A$, sural; $B$, saphenous; $C$, lateral cutaneous; $D$, iliohypogastric; $E$, median (interdigital branches) and $F$, sensory branch of the trigeminal nerve. The curves are all from the same volunteer. $S D$ of control values are indicated by interrupted lines. They all disclose a double peak facilitation whose amount does not depend on the proximity of the stimulated nerve.

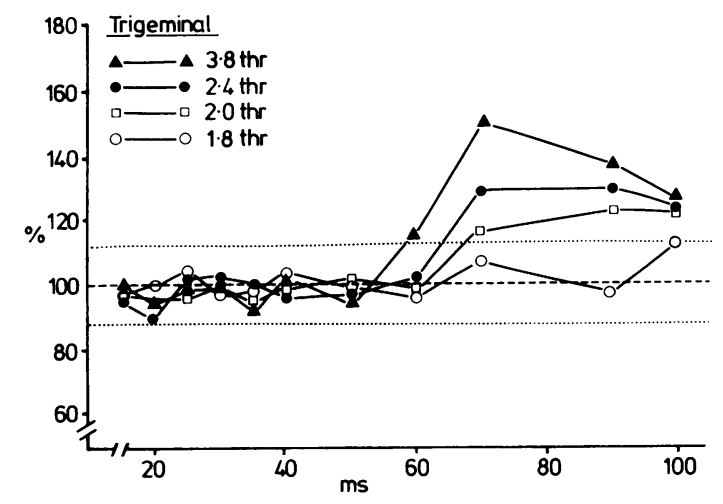

Fig 2 Amplitude changes of the soleus $H$ reflex following stimulation of the first division of the ipsilateral trigeminal nerve at various intensities. SD of control values are indicated by interrupted lines. Facilitation appears for a stimulus intensity of $2 \times$ the perception threshold and increased progressively with stimulation at 2.4 and 3.8 times sensory threshold. This latter is painful. 
(a)

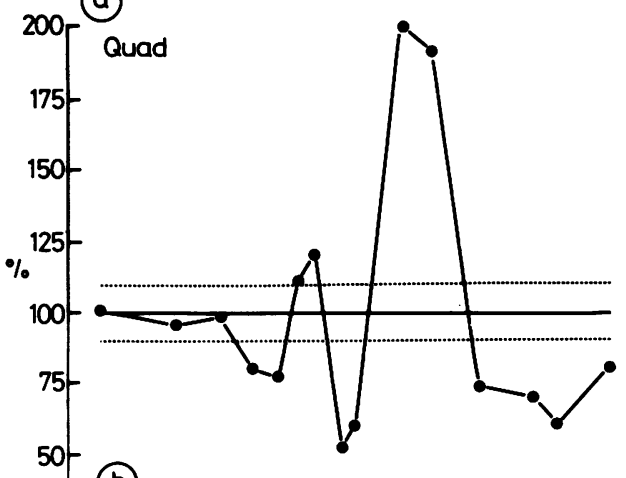

(b)

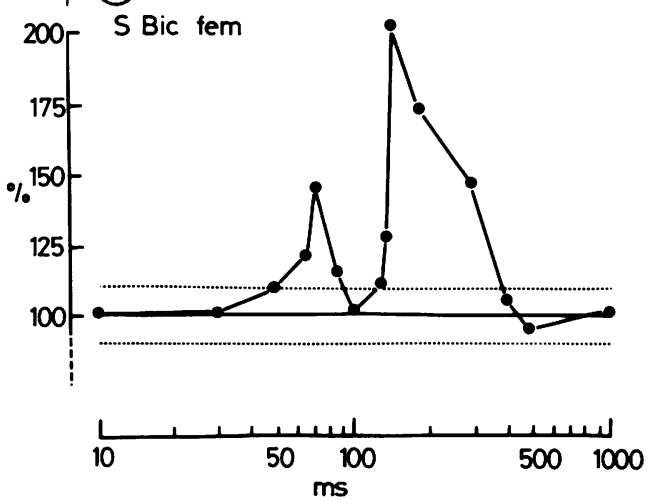

Fig 3 Excitability curves of the tendon reflexes of two antagonistic motor nuclei of the thigh, the quadriceps femoris $(A)$ and the short biceps femoris $(B)$ respectively. The conditioning stimulation is applied on the contralateral sural nerve. $S D$ of control values are indicated by interrupted lines.



Fig 4 Effects of ipsilateral sural nerve stimulation upon the amplitude of the tendon jerks of $(A)$ the biceps brachii and $(B)$ triceps brachii respectively. Oscillograms (upper part) illustrate the changes in amplitude at various delays. The excitability curves (lower part) show a multiphasic facilitation which appears in phase on 2 antagonistic motor nuclei. SD of control values are indicated by interrupted lines. 
hand, the intensity of facilitation is similar whatever sensory nerve is stimulated with the exception of the lateral cutaneous nerve of the thigh, which regularly elicits more marked facilitation.

\section{b. EXCITABILITY CHANGES IN VARIOUS MOTOR NUCLEI FOLLOWING SURAL NERVE STIMULATION}

Figure 3 shows excitability changes in two functionally antagonistic motor nuclei, the quadriceps and the short biceps, following stimulation of the sural nerve at moderate intensity $(2.7 \times \mathrm{Thr})$. As in fig 1 , two peaks of facilitation were observed. These results are very similar to those previously reported ${ }^{1}$ on the soleus and tibialis anterior motor nuclei. Thus sural nerve stimulation modulates the excitability of motor nuclei of thigh as well as of calf muscles.

We later investigated the effect of sural nerve stimulation $(3 \times \mathrm{Thr})$ on the excitability of motor nuclei in the cervical enlargement. This was done by examining the excitability curves of the biceps and triceps brachii; fig 4 shows a typical example of the results. There is a first peak of facilitation whose onset is seen after $35-45 \mathrm{~ms}$. This is less than the latency of the first peak recorded in the soleus itself if account is taken of the latency differences of the two reflexes under test: $30 \mathrm{~ms}$ for the soleus and 16 $\mathrm{ms}$ for the biceps brachii. Subsequently, the conditioned biceps curve exhibits a discrete inhibition after a delay of $70 \mathrm{~ms}$, followed by a second peak of facilitation culminating at $90 \mathrm{~ms}$. This peak declines and is sometimes followed by a third phase of facilitation which peaks between 180 and $200 \mathrm{~ms}$. It can be seen that the facilitation curve has the same appearance in the two antagonistic motor nuclei. The first phase of facilitation is often less marked than the second, and indeed is sometimes lacking. In such cases, the first peak of facilitation reaches its maximum after the usual latency of $90 \mathrm{~ms}$. The effect on the brachial motor nuclei appears at stimulus intensities of $3 \times$ Thr. When the stimulation intensity is increased and becomes painful $(5 \times$ $\mathrm{Thr}$ ), there is no increase in facilitation.

Finally, we investigated the effect of sural nerve stimulation $(3 \times \mathrm{Thr})$ on the excitability of the motor nucleus of the masseter (fig 5). The figure illustrates the average of experiments carried out on seven subjects. Following stimulation systematic variations in excitability are once again observed. Several phases of facilitation (frequently three) with onsets at 32, 69 and $96 \mathrm{~ms}$ were observed. Important variations in the amplitude of the first peak of facilitation were observed: while it was strongly marked after the first stimulations of a series where the same conditioning was used, it rapidly became reduced as the stimuli were repeated. In order to
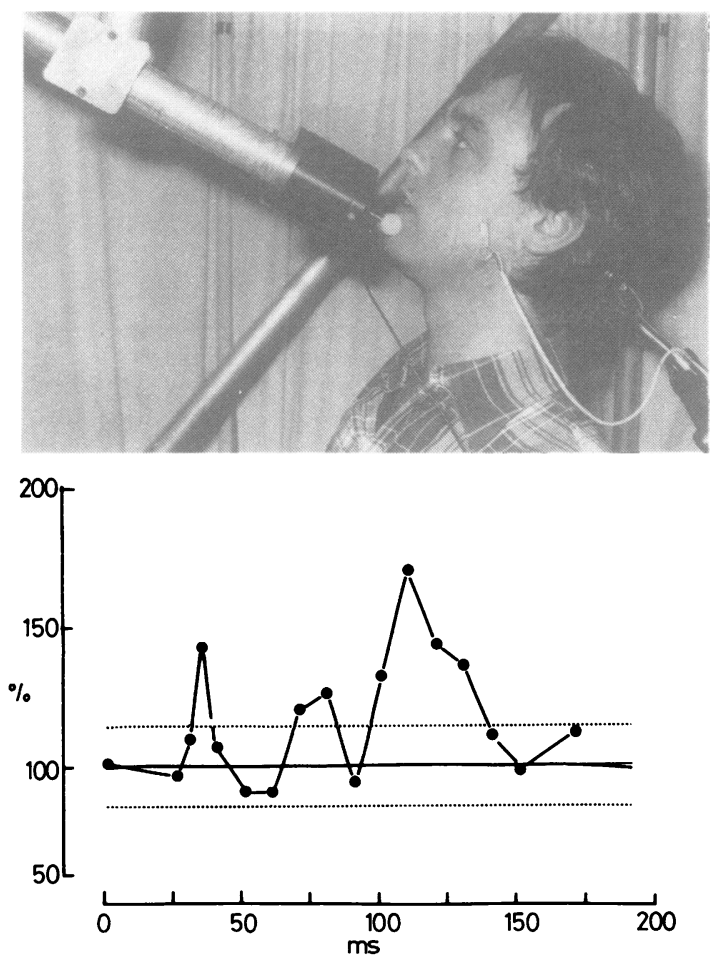

Fig 5 The upper part illustrates how the masseter reflexes are elicited. The lower part shows the mean excitability curve (7 subjects) of the masseter reflex after stimulation of the sural nerve. Standard deviations are indicated by interrupted lines.

demonstrate this peak, random stimulation is necessary, as well as short stimulus series; alternatively, only the first responses of a series are retained. It is thus evident that this phase is labile and habituates rapidly.

\section{Discussion}

In the first place, our results indicate that the cutaneous receptive field from which stimulation can influence the excitability of the soleus motor nucleus is very extensive, and in fact covers the whole body-upper and lower limbs, trunk and head. Whatever nerve outside the lower limb (where the test is carried out) is stimulated, the effect consists essentially of a facilitation most often exhibiting two successive and distinct phases which we have called $\mathrm{F}(\mathrm{a})$ and $\mathrm{F}(\mathrm{b})$. Furthermore, stimulation of a given nerve gives rise to two phases of facilitation in lower limb motor nuclei, and two or more often three phases of facilitation in upper limb or head motor nuclei. The similarity of results is also seen with 
respect to the amplitude of facilitation, which does not become attenuated with increasing distance from the motor nucleus tested. Thus excitability changes in a motor nucleus are not related to the proximity of the segment of entry of the conditioning afferents into the cord. Studying changes in the electromyogram, Meier-Ewert $e t a^{2}$ and PiesiurStrehlow and Meinck $^{3}$ have also shown that exteroceptive stimuli acted at a distance and did not have an effect limited to a few spinal segments close to the point of entry of the afferents into the cord. The method of excitability curves, which has previously been used in a few instances, ${ }^{47}$ makes it easier to quantify effects and measure delays.

The afferents which are effective in bringing about facilitation are of low-medium threshold because they are activated at intensities ranging between $2 \times \mathrm{Thr}$ (see fig 2 ) to about $3 \times \mathrm{Thr}$. Stronger stimuli increase the amplitude of facilitation, but the recruitment of small fibres which carry painful sensation did not significantly affect the results observed.

Facilitation occurred simultaneously in muscles with mutually antagonistic functions, such as the quadriceps and the short head of biceps femoris (fig 3 ) or the biceps and triceps brachialis (fig 4). Distant effects were seen equally in rostro-caudal (fig 1) and caudo-rostral directions (figs 3-4 and 5). They could even be observed in the brainstem, thus indicating that coordination mechanisms between upper and lower limbs do not suffice to explain the observations. $^{5}$ Actually, if comparison is limited to upper versus lower limb cutaneous effects on the soleus nucleus, a direct interlimb pathway could be postulated to interpret latencies differences in facilitation (after subtraction of peripheral conduction times, facilitation from the lateral cutaneous nerve of the thigh supervenes earlier than that from the median nerve). Such a hypothesis has been proposed. ${ }^{7}$ However, this explanation is more difficult to maintain if results observed after trigeminal nerve stimulation are taken into account. The facilitation brought about by this latter appears some $10 \mathrm{~ms}$ before that induced by ileohypogastric nerve stimulation although the distance between trigeminal nerve and soleus motor nucleus is $45 \mathrm{~cm}$ longer than that between ileohypogastric nerve and the same nucleus. These facts lead thus to postulate another explanation than interlimb facilitation. Moreover interlimb facilitation cannot be the only factor as can be seen by comparing the effects which follow stimulation of a single nerve (the sural) when correction has been made for the latencies of various reflexes in order to study the sequence of facilitations within the spinal cord (by adding half the latency of the test reflex). The first phase of facilitation, which has a similar threshold, mean amplitude, and duration in all motor nuclei explored, appears first in the masseter $(32+5 \mathrm{~ms}$ correction factor), then in the arm muscles $(35+8 \mathrm{~ms}$ for biceps, $45+8 \mathrm{~ms}$ for triceps) and finally in lower limb muscles $(47+10$ $\mathrm{ms}$ for biceps femoris, $56+10 \mathrm{~ms}$ for quadriceps, $56 \cdot 5+15 \mathrm{~ms}$ for soleus). This phenomenon is currently being studied, and will be the subject of a later publication.

In order to explain the results reported in the present study, though not all of the excitability changes in the soleus motor nucleus following high-intensity stimulation of the sural nerve, it is insufficient to consider only segmental spinal mechanisms, ${ }^{46}$ or reciprocal effects between cervical and lumbo-sacral segments. ${ }^{578}$ Indeed it is tempting to hypothesise in addition the existence of a supraspinal centre which is activated by medium-low-threshold exteroceptive afferents and which facilitates all motor nuclei in a rostro-caudal sequence, and is responsible for certain features of the first and second peaks. Recent data from Jenner and Stephens, ${ }^{9}$ providing strong evidence of cutaneous reflex components mediated through long-loop circuits in man' support this view. The facilitation latencies reported in the present study would be compatible with such a mechanism.

Following this line of reasoning, a parallel may be drawn with the startle reaction which follows (for example) a brief but intense auditory stimulus. In this instance, the facilitation is also nonhomogeneous and may exhibit two phases. The rapid habituation of facilitation observed in both situations is also analogous. Thus, if it be postulated that facilitation of motor nuclei depends, at least in part, on the activation of a supraspinal structure, it does not appear necessarily to be the case that this mechanism is specific, activated only be cutaneous stimuli. The short duration of the effective stimulation and the element of surprise related to random stimulation also deserve consideration.

\section{References}

${ }^{1}$ Delwaide PJ, Crenna P, Fleron MH. Cutaneous nerve stimulation and motoneuronal excitability: I, soleus and tibialis anterior excitability after ipsilateral and contralateral sural nerve stimulation. J Neurol Neurosurg Psychiatry 1981;44:699-707.

${ }^{2}$ Meier-Ewert K, Schmidt C, Nordmann G, Humme U, Dahm J. Averaged muscle-response to repetitive sensory stimuli. In: JE Desmedt, ed. New Developments in Electromyography and Clinical Neurophysiology. Basel: Karger 1973, vol. 3, 767-72.

${ }^{3}$ Piesiur-Strehlow B, Meinck HM. Response patterns of human lumbosacral motoneurone pools to distant somatosensory stimuli. Electroencephalogr. Clin Neurophysiol 1980;48:673-86. 
${ }^{4}$ Gassel MM, Ott KH. An electrophysiological study of the organisation of innervation of the tendon jerk in humans. In: JE Desmedt, ed. New Developments in Electromyography and Clinical Neurophysiology Basel: Karger 1973, vol. 3, 308-17.

${ }^{5}$ Kearney R, Chan CW. Interlimb reflexes of human arm muscles to cutaneous stimulation of the foot. Brain Res 1979;170:214-17.

${ }^{6}$ Hugon M. Exteroceptive reflexes to stimulation of the sural nerve in normal man. In. JE Desmedt, ed. New Developments in Electromyography and Clinical
Neurophysiology Basel: Karger 1973, vol. 3, 713-29.

${ }^{7}$ Meinck HM, Piesiur-Strehlow B. Reflex evoked in leg muscles from arm afferents: a propriospinal pathway in man? Exp Brain Res 1981,43:78-86.

${ }^{8}$ Kots, Ya M. The Organisation of Voluntary Movement. Neurophysiological mechansims New York: Plenum Press 1977, p. 189.

9 Jenner, Jr, Stephens JA. Cutaneous reflex responses and their central nervous pathways studied in man. $J$ Physiol (Lond) 1982;333:405-19. 